\title{
Energy spectra of cosmic ray heavy nuclei in the NUCLEON space experiment after two years of data acquisition
}

\section{A. Panov*}

Skobeltsyn Institute of Nuclear Physics, Moscow State University, Moscow, Russia

E-mail: panovedec1.sinp.msu.ru

\section{E. Atkin}

National Research Nuclear University "MEPhI", Moscow, Russia

E-mail: evatkin@mephi.ru

\section{N. Gorbunov}

Joint Institute for Nuclear Research; Dubna State University, Dubna, Russia

E-mail: gorbunovesunse.jinr.ru

\section{Grebenyuk}

Joint Institute for Nuclear Research; Dubna State University, Dubna, Russia

E-mail: grebenejinr.ru

\section{Karmanov}

Skobeltsyn Institute of Nuclear Physics, Moscow State University, Moscow, Russia E-mail: karmanov68@mail.ru

\section{Kovalev}

Skobeltsyn Institute of Nuclear Physics, Moscow State University, Moscow, Russia E-mail: im.kovalev@physics.msu.ru

\section{Kudryashov}

Skobeltsyn Institute of Nuclear Physics, Moscow State University, Moscow, Russia E-mail: ilya.kudryashov.85@gmail.com

\section{A. Kurganov}

Skobeltsyn Institute of Nuclear Physics, Moscow State University, Moscow, Russia E-mail: meesx107.ru

\section{Merkin}

Skobeltsyn Institute of Nuclear Physics, Moscow State University, Moscow, Russia E-mail: merkinmesilab.sinp.msu.ru

\section{Podorozhny}

Skobeltsyn Institute of Nuclear Physics, Moscow State University, Moscow, Russia E-mail: dmp@eas.sinp.msu.ru

\section{S. Porokhovoy}

Joint Institute for Nuclear Research, Dubna, 141980, Russia

E-mail: porokhenusun. jinr.ru 


\section{Shumikhin}

National Research Nuclear University “MEPhI”, Moscow, Russia

E-mail: shuma.v.v@mail.ru

\section{A. Tkachenko}

Joint Institute for Nuclear Research, Dubna, Russia

E-mail: avtajinr.ru

\section{Tkachev}

Joint Institute for Nuclear Research; Dubna State University, Dubna, Russia

E-mail: tkatchevejinr.ru

\section{A. Turundaevskiy}

Skobeltsyn Institute of Nuclear Physics, Moscow State University, Moscow, Russia

E-mail: ant@eas.sinp.msu.ru

\section{O. Vasiliev}

Skobeltsyn Institute of Nuclear Physics, Moscow State University, Moscow, Russia E-mail: oav@rsx.sinp.msu.ru

\section{A. Voronin}

Skobeltsyn Institute of Nuclear Physics, Moscow State University, Moscow, Russia

E-mail: voroninesilab.sinp.msu.ru

The energy spectra of abundant heavy nuclei from carbon to iron collected by the NUCLEON space experiment after two years of operation are presented. The spectra were measured with use of both calorimeter and KLEM methods. The complex behavior of the spectra from carbon to silicon in the energy range from a few $\mathrm{TeV}$ to hundreds of $\mathrm{TeV}$ per particle is presented and discussed. The difference between the spectrum of iron and the spectra of other heavy nuclei from carbon to silicon and a signature of a universal break in the spectra of all nuclei near the magnetic rigidity $10 \mathrm{TV}$ are emphasized. Some new features in the stectra of heavy nuclei are discussed.

35th International Cosmic Ray Conference - ICRC2017

10-20 July, 2017

Bexco, Busan, Korea

\footnotetext{
*Speaker.
} 


\section{Introduction}

One of the most notable features in the energy spectrum of cosmic rays is the sharp increase in the slope of the energy spectrum near $3 \times 10^{15} \mathrm{eV}(3 \mathrm{PeV})$ per particle - the so-called "knee." The nature of this "knee" is still unclear, and represents one of the major mysteries of cosmic ray physics and space physics in general. The "knee" in the spectrum of cosmic rays has been found and is still observed in the EAS (extensive air showers) experiments, which provide data on the energy spectrum of cosmic rays at very high energies, but do not give reliable information about their chemical composition. At the same time, for understanding the physics near the "knee," it would be very important to know the behavior of the individual components of the flux of cosmic rays near this area. Much more detailed information on the chemical composition of cosmic rays is provided by so-called direct experiments, in which the spectrometer is moved out of the atmosphere, where cosmic-ray particles can be observed directly, using different types of spectrometers. One of the main tasks for modern cosmic-ray direct experiments is the study of chemical composition near the knee region.

Even in the energy region below $1 \mathrm{PeV}$ per particle there are signatures of complex behavior of cosmic-ray spectra. We can mention observation of hardening of the spectra of heavy nuclei near the energy $\sim 10 \mathrm{TeV}$ per particle that corresponds to the energy of a few hundred GeV per nucleon observed in ATIC and CREAM experiments [1,2]. The details of behavior of the heavy nuclei spectra at energies higher than $100 \mathrm{TeV}$ per particle are almost completely unknown and might contain interesting features. In this paper we present the latest data of the direct NUCLEON space experiment on spectra of abundant heavy nuclei up to the energies $\sim 200 \mathrm{TeV}$ per particle.

\section{The instrument and data taking}

The NUCLEON space experiment was designed mainly to measure the spectra of cosmic ray nuclei with an individual charge resolution in the energy range from a few $\mathrm{TeV}$ to $1 \mathrm{PeV}$ per particle, while having a lower energy threshold of a few hundred GeV. The most important feature of the NUCLEON detector is the implementation of two different particle energy measurement methods: the first uses an ionization calorimeter, and the second is a kinematic method, the Kinematic Lightweight Energy Meter (KLEM) [3, 4, 5, 6, 7], which is based on the measurement of the multiplicity of secondary particles after the first nuclear interaction of a primary particle with a target of the spectrometer. The advantage of the KLEM method compared to the conventional calorimetric method is the ability to provide a high aperture of the device with a low weight of the equipment. The presence of the two methods of energy measurement in the NUCLEON detector will allow studying and calibrating the new KLEM method using a conventional calorimetric method.

The main systems of the spectrometer are four of planes of the charge measurement system (ChMS), a carbon target, six planes of the energy measurement system using the KLEM method (KLEM system tracker), three double-layer planes of the scintillator trigger system, and a small aperture calorimeter (IC). Details of the detector design are provided in the articles [8, 9, 10, 11, 12]. The weight of the detector is approximately $360 \mathrm{~kg}$; the power consumption does not exceed $160 \mathrm{~W}$. On December 28, 2014, the NUCLEON detector was launched into a sun-synchronous orbit with an average altitude of $475 \mathrm{~km}$ and an inclination of 97 degrees as an additional payload of the Russian 
satellite Resource-P 2. On January 11, the NUCLEON detector was powered and started to collect data. The planned lifetime of the NUCLEON detector is at least five years. Some details of the implementation of methods of the energy measurement with KLEM and calorimetric methods in the NUCLEON experiment are given in the paper [13].

In this report we present and discuss the data related to the abundant heavy nuclei from carbon to iron for period of data taking of the NUCLEON experiment from July 2015 to the end of June 2017. There were 334 days of pure astronomical time of the data taking that correspond to 218 days of the apparatus live time. There was eight months delay in data acquisition in 2015-2016 due to the solution of the main task of the Resource-P 2 serial satellite was incompatible with the operation of the NUCLEON observatory as an additional payload.

\section{Results and discussion}

Figure 1 shows the energy spectra of carbon and oxygen nuclei obtained by the NUCLEON experiment both for the KLEM and calorimeter methods. There are no strong deviations from the results of the other experiments (see the caption under the pictures). Some differences between the calorimeter and the KLEM methods are seen for the carbon spectrum, but for oxygen spectrum the data of KLEM and calorimeter are in good agreement. If the differences in carbon data would be systematic, than one should expect similar differences in the oxygen spectrum, but actually it is not the case. Therefore we can not confidently relate the differences of the results in the carbon spectrum to a systematic origin; the origin of this discrepancy has been studied.
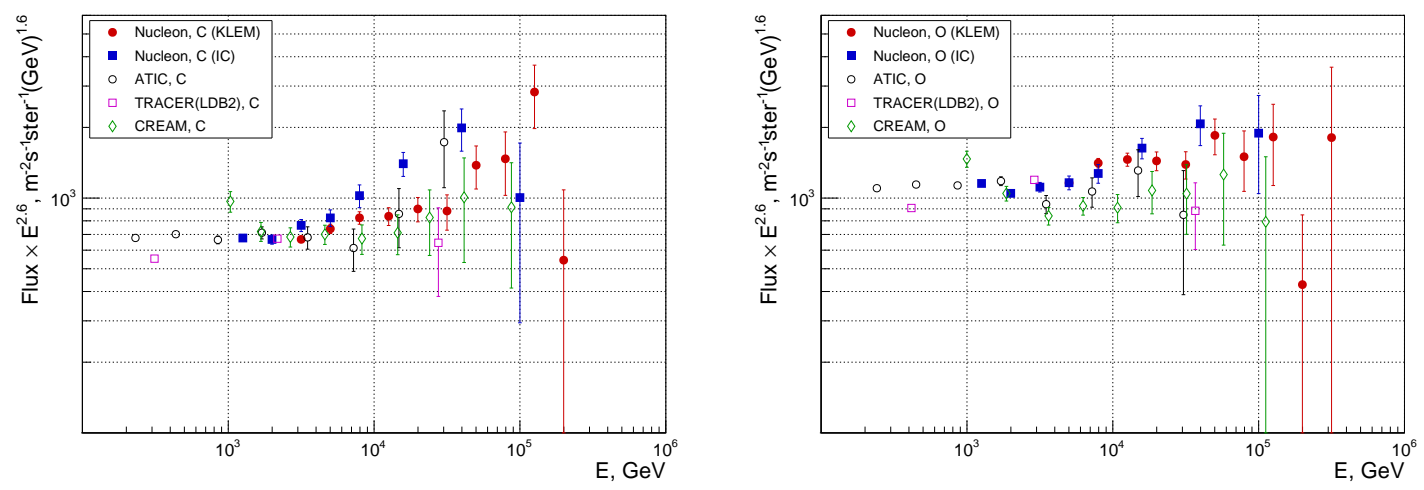

Figure 1: Energy spectra of carbon and oxygen nuclei obtained by the NUCLEON experiment and in the experiments ATIC [14], TRACER(LDB2) [15], and CREAM [2].

The spectra of carbon and oxygen are hard above energy $2 \mathrm{TeV}$ per particle. From the KLEM data $\gamma_{\mathrm{C}}=2.381 \pm 0.033, \gamma_{\mathrm{O}}=2.442 \pm 0.039$; from the calorimeter data $\gamma_{\mathrm{C}}=2.269 \pm 0.042, \gamma_{\mathrm{O}}=$ $2.410 \pm 0.035$. It is seen that the spectrum of carbon may be harder than the spectrum of oxygen, but the statistical significance of this conclusion is not high: $1.2 \sigma$ and $2.6 \sigma$ for the KLEM and calorimeter spectra respectively.

Figure 2 shows the ratio of the carbon spectrum to the oxygen spectrum in the terms of energy per nucleon. A sign of more hard spectrum of carbon also seen in this ratio, but the statistical significance is not high again. A difference of the slopes of the carbon and oxygen spectra may be 


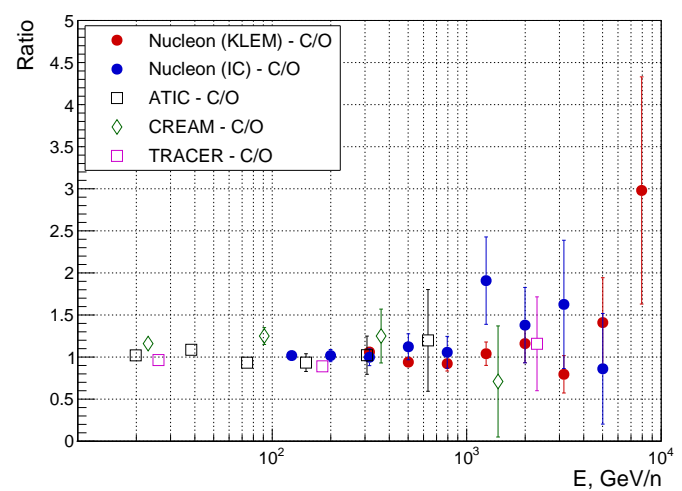

Figure 2: Energy spectra of carbon and oxygen nuclei obtained by the NUCLEON experiment and in the experiments ATIC [14], CREAM [2], and TRACER(LDB2) [15].

important for understanding of mechanism of cosmic ray acceleration since currently all models predict essentially same slopes for carbon and oxygen up to very small propagation effects.

Figure 3 shows the spectra of $\mathrm{Ne}, \mathrm{Mg}$, Si. The spectral indices of the spectra obtained for the calorimeter method are respectively: $\gamma_{\mathrm{Ne}}=2.391 \pm 0.046, \gamma_{\mathrm{Mg}}=2.544 \pm 0.041, \gamma_{\mathrm{Si}}=2.536 \pm$ 0.038. The spectrum of $\mathrm{Ne}$ is harder than the spectra of $\mathrm{Mg}$ and $\mathrm{Si}$ with statistical significance $2.5 \sigma$ and $2.4 \sigma$ respectively. The linear fit of the nuclei charge - spectral index relation shows (Figure 3, the bottom right plot) the trend of the slope of spectra in the group Ne-Mg-Si exist with the statistical significance $2.3 \sigma$. The KLEM method produces lower significance of $\mathrm{Ne}-\mathrm{Mg}$-Si data because of higher energy threshold of the KLEM method for very heavy nuclei in the comparison with the calorimeter method, so the KLEM data do not considered here.

Figure 4 shows the spectrum of iron in the terms of energy per particle and the ratio of the spectrum of all nuclei $6 \leq Z \leq 14$ to the spectrum of iron in the terms of energy per nucleon. The spectral index of the iron spectrum from the calorimeter data is $\gamma_{\mathrm{Fe}}=2.523 \pm 0.023$. Generally, the spectrum of iron is steeper than the spectra of more light abundant heavy nuclei. The spectrum of iron is steeper than the spectra of carbon and oxygen with the statistical significance $5.0 \sigma$ and $2.7 \sigma$ respectively. The ratio of the spectrum of all nuclei $6 \leq Z \leq 14$ to the spectrum of iron shows qualitatively more steeper character of the iron spectrum than tithe spectra of other abundant heavy nuclei. Note, already the ATIC experiment [16] gave and indication of the same effect (see figure 4) but with low statistical reliability.

An interesting question is what is the transition from the flat spectra of carbon-silicon group to the steeper spectrum of iron. The answer may be found from the spectra of nuclei with intermediate charges, $\mathrm{S}$ and $\mathrm{Ca}$. This spectra are shown in figure 5. The statistics is too low yet to draw some definite conclusions.

The spectrum of all nuclei from carbon to iron in the terms of energy per nucleon measured in the NUCLEON experiment and in the ATIC experiment [19] is shown in figure 6. It is seen that after the hard part of the spectrum below the energy $5 \mathrm{TeV} /$ nucleon there is a break of the spectrum. The magnetic rigidity of the break is approximately $10 \mathrm{TV}$ and it is very close to the rigidity of similar breaks in the spectra of protons and helium measured by NUCLEON experiment (see companion paper PoS(ICRC2017)212 in this proceedings, see also previos NUCLEON paper 

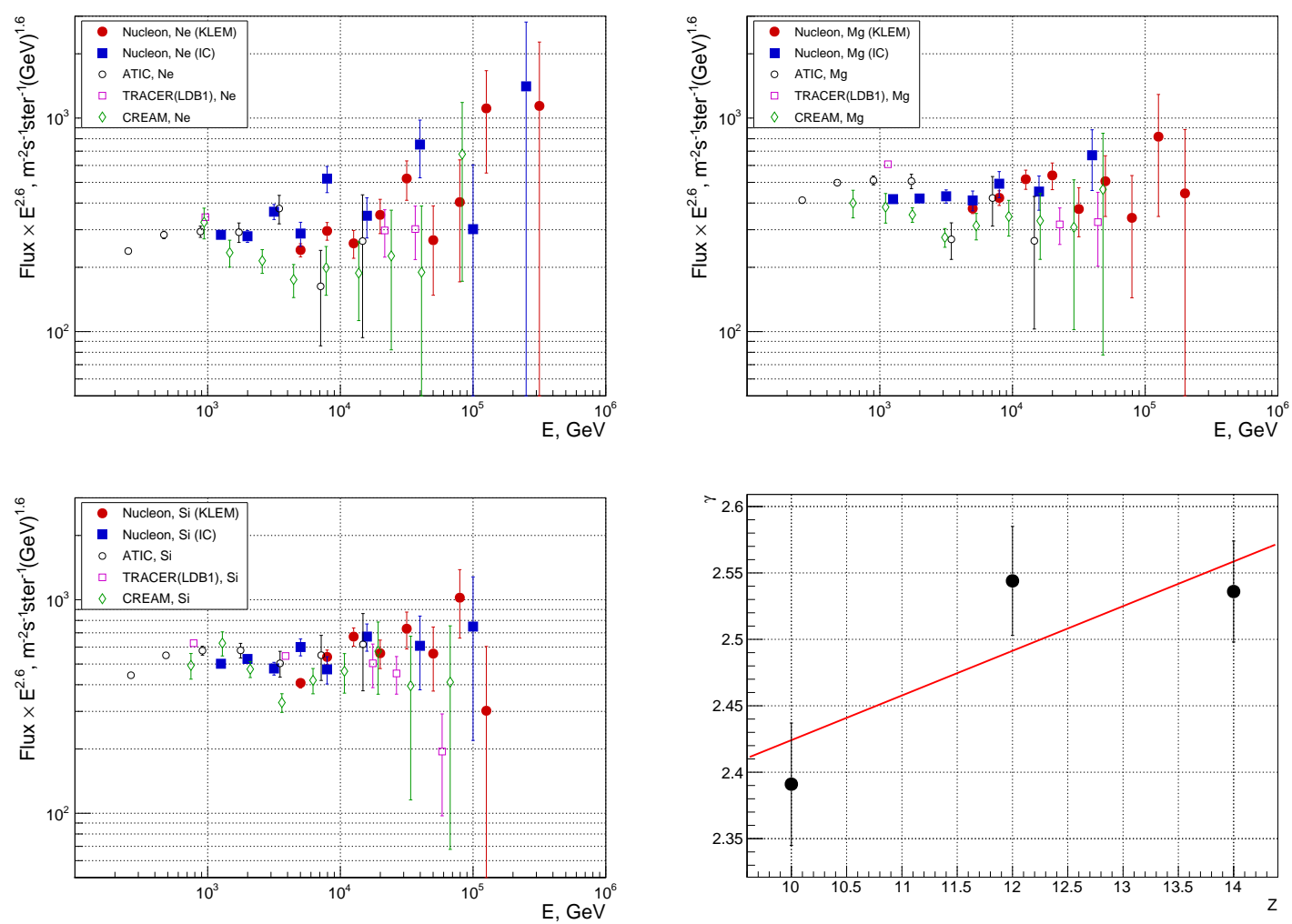

Figure 3: Energy spectra of $\mathrm{Ne}, \mathrm{Mg}$, Si obtained by the NUCLEON experiment and in the experiments ATIC [14], TRACER(LDB2) [15], and CREAM [2]. Right bottom panel: spectral index versus charge plot for $\mathrm{Ne}, \mathrm{Mg}$, Si spectra.
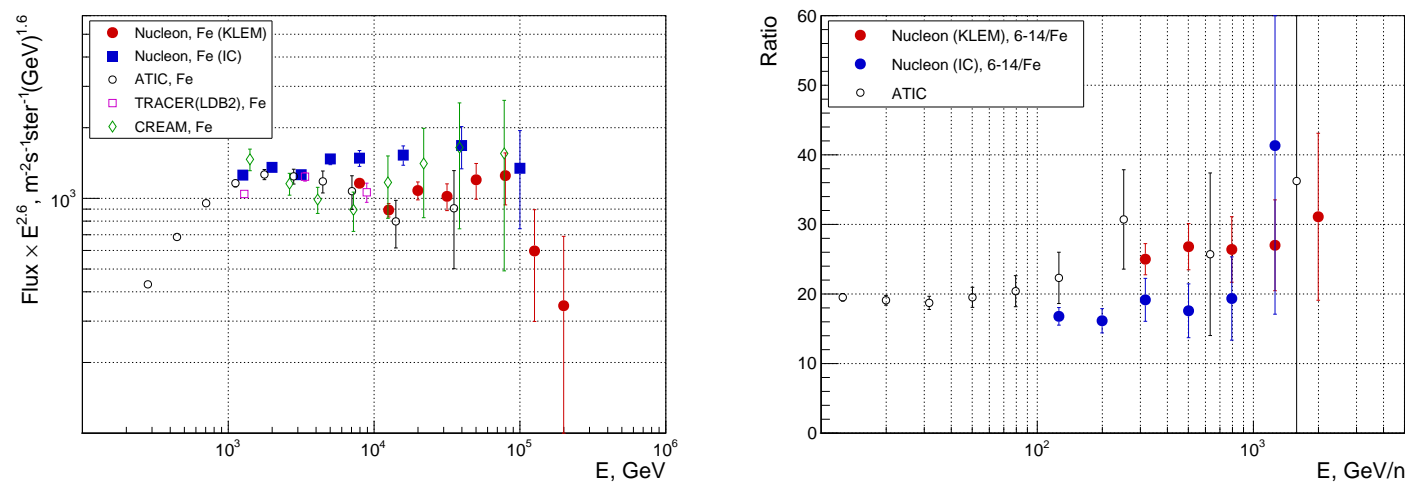

Figure 4: Left: energy spectra of iron obtained by the NUCLEON experiment and in the experiments ATIC [14], TRACER(LDB2) [15], and CREAM [2]. Right: ratio of the spectrum of all nuclei $6 \leq Z \leq 14$ to the spectrum of iron in the terms of energy per nucleon obtained by the NUCLEON experiment and by ATIC $[16]$. 

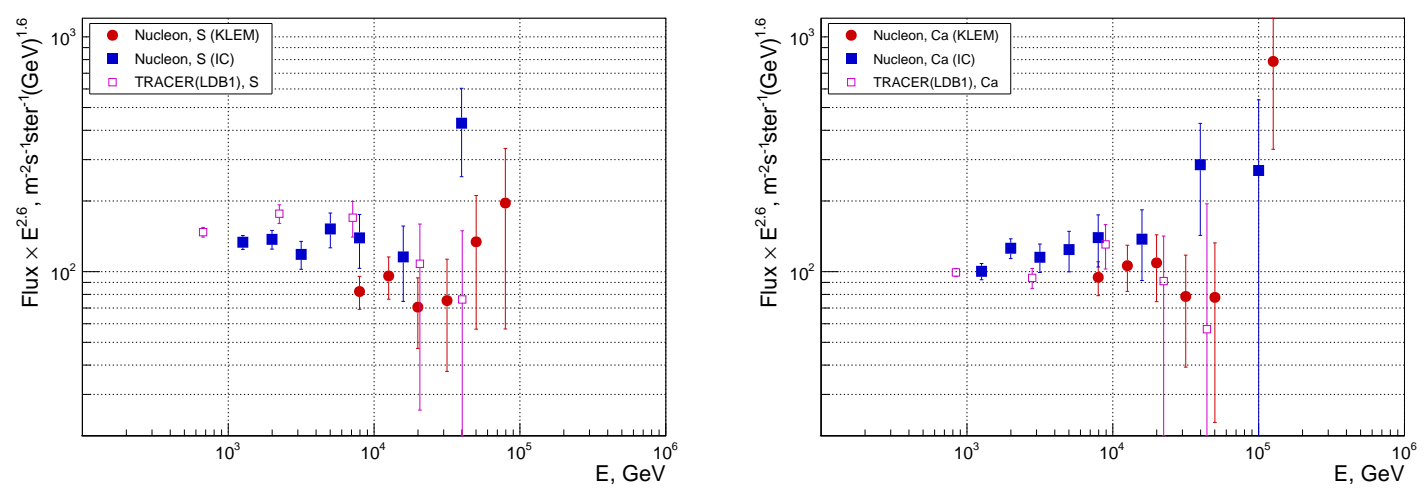

Figure 5: Energy spectra of $\mathrm{S}$ and $\mathrm{Ca}$ obtained by the NUCLEON experiment and in the experiment TRACER(LDB1) [17].

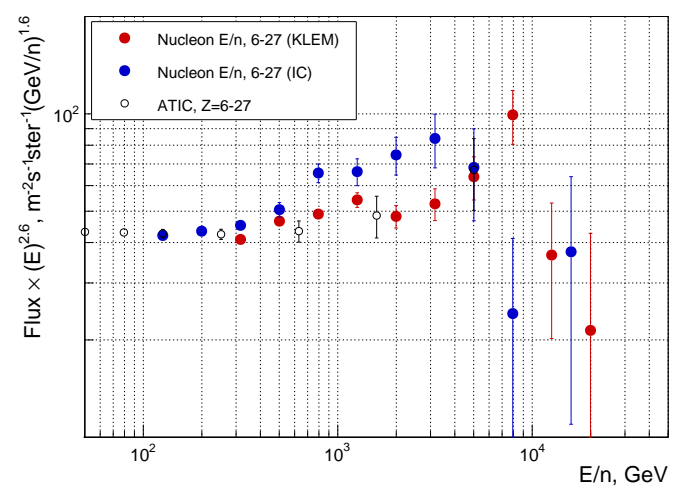

Figure 6: Spectra of heavy nuclei $(Z=6 \div 27)$ in terms of energy per nucleon from the NUCLEON experiment along with similar data from the ATIC experiment [19].

[13] and similar CREAM results [18]). This may be an indication of existence of a universal break in the spectra of all cosmic ray nuclei near the magnetic rigidity of $10 \mathrm{TV}$. In fact, the universal break near $10 \mathrm{TV}$ in all cosmic ray spectra and particularly in the spectra of heavy nuclei observed by the NUCLEON experiment was predicted by the three-component model of cosmic ray spectra of Zatsepin and Sokolskaya [20]. The break is an indication of existence of a specific type of cosmic ray sources with the maximal acceleration energy of order $10 \mathrm{TV}$ (the exact value in [20] is $50 \mathrm{TV}$, which produce visually a break in spectra near $10 \mathrm{TV}$ ). This type of sources was associated in [20] with supernova explosions into regular interstellar media (other two kinds of sources in the model were explosions of SN into super-bubble media and novae stars).

The NUCLEON experiment is in fact in its initial phase since no more than $1 / 3$ expected statistics were collected. However, the preliminary analysis of the data gives a number of indications of the existence of interesting features in the energy spectra of cosmic ray nuclei at energies from few $\mathrm{TeV}$ to $\sim 100 \mathrm{TeV}$ per particle. The statistical significance of them generally is not high yet, but the data taking is continued and the statistics increases.

We are grateful to ROSCOSMOS State Space Corporation and Russian Academy of Sciences 
for their continued support of this research.

\section{References}

[1] A. D. Panov, J. H. Adams, H. S. Ahn et al., Bulletin of the Russian Academy of Sciences: Physics 71 issue 4 (2007) 494-497, [arXiv: astro-ph / 0612377 ]

[2] H. S. Ahn, P. Allison, M. G. Bagliesi et al., ApJ Letters 714 (2010) L89-L93, [arXiv: 1004.1123 [astro-ph.HE]]

[3] J. Adams, G. Bashindzhagyan, A. Chilingaryan, et al., in AIP Conference Proceedings, vol. 504, pp. 175-180, 2000. DOI.

[4] J. Adams, G. Bashindzhagyan, P. Bashindzhagyan et al., Advances in Space Research 27 (2001) 829-831.

[5] N. A. Korotkova, D. M. Podorozhnyi, E. B. Postnikov et al., Physics of Atomic Nuclei 65 (2002) 852-860.

[6] G. L. Bashindzhagyan, A. G. Voronin, S. A. Golubkov et al., Instruments and Experimental Techniques 48 (2005) 32-36.

[7] D. M. Podorozhnyi, E. B. Postnikov, L. G. Sveshnikova and A. N. Turundaevsky, Physics of Atomic Nuclei 68 (2005) 50-59.

[8] G. Voronin, V. M. Grebenyuk, D. E. Karmanov et al., Instruments and Experimental Techniques 50 (2007) 187-195.

[9] G. Voronin, V. M. Grebenyuk, D. E. Karmanov et al., Instruments and Experimental Techniques 50 (2007) 176-187.

[10] M. Podorozhnyi, V. L. Bulatov, N. V. Baranova et al., Bulletin of the Russian Academy of Sciences 71 (2007) 500-502.

[11] V. L. Bulatov, A. V. Vlasov, N. V. Gorbunov et al., Instruments and Experimental Techniques 53 (2010) 29-35.

[12] E. Atkin, V. Bulatov, V. Dorokhov et al., Nuc. Instr. Meth. A 770 (2015) 189-196.

[13] E. Atkin, V. Bulatov, V. Dorokhov et al., Journal of Cosmology and Astroparticle Physics, 2017 issue 07 (2017) 020, [arXiv:1702.02352 [astro-ph.HE]].

[14] A. D. Panov, J. H. Adams, H. S. Ahn et al., Bulletin of the Russian Academy of Sciences: Physics 73 (2009) 564-567, [arXiv:1101.3246].

[15] A. Obermeier, M. Ave, P. Boyle, C. Höppner, J. Hörandel and D. Müller, ApJ 742 (2011) 14, [arXiv:1108.4838].

[16] A. D. Panov, N. V. Sokolskaya, and V. I. Zatsepin, Nuclear Physics B (Proc. Suppl.) 256-257 (2014) 233-240.

[17] M. Ave, P. J. Boyle, F. Gahbauer et al., ApJ 678 (2008) 262-273.

[18] Y.S. Yoon, T. Anderson, A. Barrau et al., ApJ 839 (2017) 8, [arXiv: 1704.02512 [astr-ph.HE]].

[19] A. D. Panov, V. I. Zatsepin, and N. V. Sokolskaya, Bulletin of the Russian Academy of Sciences. Physics 77 No 5 (2013) 613-615,

[20] V. I. Zatsepin and N. V. Sokolskaya, A\&Astrophysics 458 (2006) 1-5, [arXiv: 1108 . 4838]. 